\title{
Supervisory Control of Adverse Reaction of Traditional Chinese Medicine Injection
}

\author{
Jinguo Wang \\ Department of Urology \\ The First Hospital of Jilin University \\ Changchun, China \\ wangjinguolily@163.com
}

\author{
Corresponding author: Na Wang* \\ Department of Anesthesiology \\ The First Hospital of Jilin University \\ Changchun, China \\ Wangna080613@163.com
}

\begin{abstract}
Traditional Chinese medicine injections are new dosage forms of Chinese traditional medicine in China. Compare with other dosage forms such as tablets, pills and oral liquid, they have the advantage of rapid onset. Injection of the drug ingredients can rapid distribute in the target tissue or receptors, and then onset quickly. Traditional Chinese medicine injections are suitable for rescuing patients in a critical situation, intravenous drug delivery can make the drug liquid direct into the circulatory system. The traditional Chinese medicine injection has adverse reactions, so it is particularly necessary to control the quality and evaluate the safety of traditional Chinese medicine injections, and then promote the healthy development of traditional Chinese medicine injections.
\end{abstract}

Keywords-adverse reaction; traditional Chinese medicine; injection; safety

The side effects of Chinese medicine have certain threat to the safety of patients' life, so it is important to strengthen the understanding of the side effects of traditional Chinese medicine and to choose the treatment of the side effects.

Academia should clarify the definition of the side effects of traditional Chinese medicine. The clinical trials research should be strengthened. The rational use of Chinese medicine should be advocated. Thus, the side effects of traditional Chinese medicine will gradually avoided and the safety of traditional Chinese medicine can be guaranteed.

\section{The Use of Traditional Chinese Medicine InJeCtions}

The analysis of using situation and developing trend of traditional Chinese medicine injection provides the basis for rational clinical application. According to the clinical effect, Chinese medicine injection can be divided into seven types. The reasons of side effects of Chinese traditional medicine refer to the improper use, incompatibility, over dosage, individual differences and improper processing. It adjusts the side effects of factors as inappropriate usage and the correct usage. Side effects of traditional Chinese medicine include toxicity and side reactions [1]. Toxicity of traditional Chinese medicine has two meanings, in the broad and narrow sense. The toxicity of generalized meaning refers to all kinds of traditional Chinese medicine which is biased to the sick and the basic principle of traditional Chinese medicine to treat disease. Toxicity of the narrow sense refers to the pharmacological effects, therapeutic dose, poisoning dose and improper use which will cause poisoning or death.

Traditional Chinese medicine injections is new dosage forms of Chinese traditional medicine in our country, based on the traditional preparation of Chinese medicine. New technologies are introduced, for example, ultrafiltration combined with new extraction process. Compared with the traditional Chinese medicine, Chinese medicine injection are administered by intravenous drip or intramuscular injection for the treatment of disease, so the onset is fast, curative effect is significant, especially in the rescue emergency and severe cases. Other dosage forms are difficult to replace the unique role of Chinese medicine injections.

Due to the adverse reaction of traditional Chinese medicine injection in recent years, adverse reaction reports significantly increase in number. The harm of serious adverse reactions is not allowed to ignore [2]. The security risks of traditional Chinese medicine injections which are mainly reflected in the basic research are not sufficient. In our country, the medicinal material base and production technology are simple, the controllability of quality standard is poor and instructions are lack of guidance for the rational use of drugs, etc. Besides some links which deny the whole traditional Chinese medicine injection industry, problems in the traditional Chinese medicine injection have become unique clinical disease treatment methods, especially the heart and cerebrovascular disease, anti-tumor, anti-virus and some emergency treatment. It is playing an important and even an irreplaceable role. To control the quality of traditional Chinese medicine injections, promote the healthy development of traditional Chinese medicine injections, safety evaluation of traditional Chinese medicine injections is particularly important. The essential ingredients of Chinese traditional medicine in the treatment of disease are the leading cause of adverse factors. Through analysis on the studies of side effects of Chinese traditional medicine we put forward the prevention and control measures of side effects and point out that the control of traditional Chinese medicine has an important meaning which lies in promoting the modernization and internationalization of Chinese traditional medicine.

\section{SAFETY EVALUATION}

To improve safety, efficacy and quality control of traditional Chinese medicine injections, the state food and 
drug administration issued a rule to try to do a good job of safety evaluation of traditional Chinese medicine injections. It points out that safety evaluation of traditional Chinese medicine injection will be started from two aspects. One is by controlling the all links from production and selling. It will effectively control hidden troubles of traditional Chinese medicine injections. The other is the administration of the state food and drug which is partial phases for the quality control of traditional Chinese medicine injections and comprehensive evaluation. The first evaluation is for clinical use of a wide range of variety injections [3].

Tonic kinds of traditional Chinese medicine injections are widely used in the treatment of advanced malignant tumor combined with chemotherapy, for adjusting the patients' immunity and improving the patient's quality of life. However, with the wide use of tonic kinds of traditional Chinese medicine injections, the reported adverse reactions are also increasing. With the various types of traditional Chinese medicine injections, A lot of reported adverse reactions are found according to literature. Skin and systemic damage are the main clinical symptoms of adverse reaction of traditional Chinese medicine injection, which account for about $66.9 \%$ of all adverse reaction types.

To change unfavorable oral drug to injection can fast the onset and reduce gastrointestinal tract irritation. For a patient in a state of coma, convulsions and seizures, using injection is more suitable at this time. In addition, for the treatment of certain diseases, some traditional Chinese medicine injections can embody the characteristic of traditional Chinese medicine. It is because of these advantages, Chinese medicine injection volume has a big market share. Our pharmaceutical enterprises will develop the advantages of the dosage form in the future in the international market. Traditional Chinese medicine injections are step by step accepted by the world.

\section{ADVERSE REACTION}

So it is necessary to explore benefit factors like the influence of traditional Chinese medicine injection adverse reaction, so as to take corresponding intervention measures to prevent and reduce the occurrence of adverse reactions. The influence factors of adverse reaction of traditional Chinese medicine injection can be divided into three aspects, including drug quality, clinical use and the individual factor. Drug quality is the primary factor [4].

Due to the unstable quality, compound preparations, complicated composition, each flavor ingredient has as many as dozens of Chinese medicinal materials, including some unknown quantitative composition. The extraction and separation technology in the process of production has no clear technical guiding principles, so the quality control standard, storage stability of finished product in the process of transportation may change and the insoluble particles may increase. The drugs may have a certain amount of insoluble particles or other impurities. Therefore, when it is applied to patients, it is easy to cause adverse drug reactions. These needs of establishment of laws and regulations to regulate drug safety management departments and production enterprise are urgent. By improving the production technology and quality control standards the quality of medicines can be improved. The phenomenon that the clinical use of traditional Chinese medicine injection is not reasonable is the main reason that leads to adverse reactions. Irrational use mainly includes incompatibility of drugs, repeated drug use, improper use, drug overdose, contraindications and improper selection solvent, et al. The particularity of solvent also adds the incidence of adverse reactions. Intravenous drug directly into the body can stimulate the body to produce a variety of adverse reactions, especially allergic reactions [5]. Traditional Chinese medicine injection in combination with other drugs, especially multidrug share is often linked to an increased incidence of adverse reactions.

Traditional Chinese medicine plays an indispensable role in the clinical medical treatment. At the same time the doctor must pay attention that many drugs have a certain amount of biased drugs in order to realize the fundamental cure. But in clinical treatment, more and more side effects of traditional Chinese medicine clinical cases seriously threaten the life safety of patients [6].

The drug quality factor is one of the leading causes of adverse reactions. In order to get a comprehensive understanding of the influence factors of adverse reaction of Chinese medicine injection we still need to consider the drug quality factors, also can't ignore the safety hidden danger of clinical use [7].

\section{JUDGEMENT OF RESPONSIBILITY}

In adverse reactions caused by the unreasonable drug use, more effective interventions should be adopted. In the benefit type of traditional Chinese medicine injections and rational drug use, the correlation of the adverse reactions and drug quality differences need more in-depth research. The comprehensive evaluation of benefit injection and the security of clinical application of Chinese medicine promote the pharmaceutical care level and rational drug use level and reduce the incidence of adverse reactions [8].

In view of the insufficient knowledge of traditional Chinese medicine injections about safety of the public, It is one-sided understanding. Expanding propaganda changes the perception of Chinese medicine injection which claims that is safer than western medicine injection. The traditional Chinese medicine injection must use instructions issued to each department. The clinical doctor's help to correct understanding of clinical benefit and the attending of the function of the type of traditional Chinese medicine injections, dialectically use traditional Chinese medicine injection.

Correct understanding of tonic kind of traditional Chinese medicine injections and the compatibility and taboo can help the doctors to avoid the other incompatible injection mixed with traditional Chinese medicine injections after intravenous drug delivery. Correct understanding of benefit type of pharmacological effects of traditional Chinese medicine injections can avoid the super indications drug abuse.

\section{Overdose May BE HaRMful}

The traditional Chinese medicine injections have security scope. Its usage is based on injection which is equivalent to how many grams of crude drug, after calculating use dose. 
Increased doses may cause adverse consequences. Drug overdose increases the occurrence of adverse reactions [9].

\section{No DENYING THE FACT OF ADVERSE REACTION}

Things always has two sides, the Chinese medicine is not an exception. Except for the positive effects of promoting health and curative effect, there is also a breach of health, that is, negative effect of adverse reactions. Once Chinese medicine without adverse reactions and natural medicine without adverse reaction are overwhelming, the hype is to mislead the general patients for business purposes. Now the trend has been hold, but the point of view "Chinese medicine without adverse reactions" still exists in the understanding of a lot of people and should be corrected.

In spite of milder adverse reactions caused by common Chinese patent medicine than by western medicine, traditional Chinese medicine still has adverse reactions. We can form the theory basis of adverse reactions of traditional Chinese medicine from two aspects. From the theory of traditional Chinese medicine, the human body is produced by Yin and Yang which represent two kinds of material and it believes that human life can only be healthy by the relative balance of Yin and Yang. If the body appears the imbalance of Yin and Yang, it is bound to get sick. The cure is correct the imbalance of Yin and Yang. Traditional Chinese medicine treatment with traditional Chinese medicine is one of the most commonly used methods. Traditional Chinese medicine theory is that in terms of the nature and role, there is no medicine unbiased. Experts in the compendium record that no medicine is nontoxic. Biased drug can correct slant of human physiological disorders, namely therapeutic effect. If biased treatment leads to partial victory, it also can damage human body physiology which has the partial of disorder, namely adverse reactions, which fully shows that drug effect of the two sides.

Our ancestors have realized that traditional Chinese medicine is poisonous for a long time. The prescription is nontoxic. Traditional Chinese medicine explains the main reason. Traditional Chinese medicine theory of traditional Chinese medicine toxicity has been known. In the ancient time, of course, the so-called poison has many meanings. Sometimes it refers to drugs to cure disease, sometimes refers to a harm to human body, although it is the meaning of toxic drugs, these all are not synonymous with modern adverse drug reactions. There is still the possibility of consistence with modern adverse drug reaction.

\section{SUMMARY}

The specification of drug use is the basis for guiding clinical rational treatment. Updating safety information in drug specifications can timely remind relevant personnel to pay attention to the safety of the drug and minimize adverse reactions. It is recommended to timely modify the supplementary specification. It suggested that clinical medication carefully before asking allergy and allergic disease history. In the use of tonic kind of traditional Chinese medicine injections, it is important to minimize the merger kinds. For special groups including old patients and patients with allergic history, it is sensitive to drug reaction of patients to do medicine allergic test, minimize the dangers of adverse reaction of patients. More importantly, clinicians should be strict in accordance with the drug instructions of function indications, usage and dosage to avoid unreasonable drug use and to take comprehensive intervention measures in many aspects.

\section{ACKNOWLEDGEMENT}

This research was financially supported by the First Hospital of Jilin University.

\section{REFERENCES}

[1] Li Zhang, Jing Hu, Lu Xiao, Yongling Zhang, Wei Zhao, Wenke Zheng, Hongcai Shang. Adverse drug reactions of Shenmai injection: a systematic review. Journal of Evidence-Based Medicine. 2010

[2] Rajewski R A, Traiger G, Bresnahan J, Jaberaboansari P, Stella V J, Thompson D O. Preliminary safety evaluation of parenterally administered sulfoalkyl ether beta-cyclodextrin derivatives. Journal of Pharmaceutical Sciences. 1995

[3] Corsonello Andrea, Pedone Claudio, Corica Francesco, Mazzei Bruno, Di Iorio Angelo, Carbonin Pierugo, Incalzi Raffaele Antonelli. Concealed renal failure and adverse drug reactions in older patients with type 2 diabetes mellitus. The journals of gerontology. Series A, Biological sciences and medical sciences. 2005

[4] Wen-Yuan Du, Cheng Chang, Yu Zhang, Yu-Ying Liu, Kai Sun, ChuanShe Wang, Ming-Xia Wang, Yuan Liu, Fu Wang, Jing-Yu Fan, PengTao Li, Jing-Yan Han. High-dose chlorogenic acid induces inflammation reactions and oxidative stress injury in rats without implication of mast cell degranulation[J]. Journal of Ethno pharmacology. 2013

[5] Long Chen, Tom Titch, Zhuoka Luo, Yi Xu, Xuehua Li, Feifei Huang, Lei Liu, Wei Xiao, Hao Wu, Zhongyue Wang. Confirmation of a proarrhythmic risk underlying the clinical use of common Chinese herbal intravenous injections[J]. Journal of Ethno pharmacology. 2012

[6] Li Zhang, Jing Hu, Lu Xiao, Yongling Zhang, Wei Zhao, Wenke Zheng, Hongcai Shang. Adverse drug reactions of Shenmai injection: a systematic review[J]. Journal of Evidence-Based Medicine. 2010

[7] Li Wang, Lan Cheng, Qiang Yuan, Xiaohua Cui, Hongcai Shang, Boli Zhang, Youping Li. Adverse drug reactions of Shuanghuanglian injection: A systematic review of public literatures[J]. Journal of Evidence - Based Medicine. 2010

[8] Kunmei Ji, Jiajie Chen, Meng Li, Zhigang Liu, Lixin Xia, Chunbo Wang, Zhengke Zhan, Xuli Wu. Comments on serious anaphylaxis caused by nine Chinese herbal injections used to treat common colds and upper respiratory tract infections[J]. Regulatory Toxicology and Pharmacology. 2009

[9] Sarah Gould, Robert C. Scott. 2-Hydroxypropyl- $\beta$-cyclodextrin (HP- $\beta$ CD): A toxicology review[J]. Food and Chemical Toxicology. 2005 